\title{
Home Medication Review in Improving Patient Medication Adherence and Minimizing Medication Wastage among Type 2 Diabetes Patients
}

\section{Fahad Saleem*, Mohamed Azmi Hassali and Ee Pin Chow}

School of Pharmaceutical Sciences, Universiti Sains Malaysia, Malaysia

\section{Short Commentary}

In Malaysia, public primary health care clinic serve as the main entry point for most of the chronic patients including diabetes and hypertension patients. The increasing complexity of patients and their treatment regimens need providers who can manage their medication therapy, drug-related problems and identify adverse drug reactions. Unfortunately with a long interval between patients visit and time constrain during the clinic day, the healthcare providers facing difficulties in providing optimal care to those patients [1]. In most cases, physicians and pharmacists received insufficient information on what medications especially the over-the counter medications that patients take at home [2]. In this context, patients need more contact with the healthcare team rather than a single provider in the primary healthcare [1].

In Malaysia, the role of pharmacists have transformed from dispensing to clinical in helping physician to review patients' medication in the ward. Slowly, the pharmacists' roles have been extended to Medication Therapy Adherence Clinics (MTAC). These MTACs are conducted by pharmacists in collaboration with physicians and other healthcare providers to help patients in improving their quality use of medications. However, during the vulnerable period after discharge from hospital or after the monthly follow up in primary care clinics, medication errors or potential medication errors may be present without the knowledge of the physician. To be able to understand in depth the behavioral of patients taking their medications and the storage condition of the medications at home, a home medication review is needed.

Therefore, a non-clinical, randomized trial was carried out in Klinik Kesihatan Bukit Minyak, Seberang Perai from April 2013 until March 2014. Eligible patients were randomized into 2 groups; Usual care (UG) and Home Based Intervention (HBI) by using a coin toss approach. Patients' baseline clinical parameter such as HbAlc, blood pressure, $\mathrm{BMI}$, fasting blood sugar were collected during the recruitment. Each patient in the HBI arm was visited twice at his or her houses. During the first visit, the pharmacist did blood pressure monitoring and assessed patients' adherence to medication by using validated questionnaire $[3,4]$.Patients were interviewed to identify any drug-related problems. Pharmacist rectified the problems and discussed with the physician on the regimen if needed. The storage of patients' medications at the house was also checked. Counseling was given to the patients as well as the caregivers on the disease itself, the proper use of medications and the storage of medications. During the second visit (which is 4-6 weeks after the $1^{\text {st }}$ visit), patients' blood pressure was monitored and pharmacist reassessed patients' adherence using the same validated questionnaire. Excessive or balance medications was collected back. Any new drug-related problems were identified during the second visit. Medication storage was checked back again. During the second visit, patients were asked a few questions regarding their perception on the Home Medication Review Program.

Out of 158 patients, and the 150 patients who consented to participate, 75 were allocated to the HBI group while 75 were allocated to the UC group. Women were over-represented in the entire cohort $(95,63.33 \%)$, and majority of the respondents were categorized under the $>55$-year-old group, with a mean age of $60.31 \pm 9.56$ years. At baseline, diabetes-related knowledge and medication adherence were reported as low in both HBI $(4.19,3.53)$ and UC $(4.6,3.49)$ groups. Furthermore, poor glycaemic control was reported in both HBI and UC (8.92 and 9.23) groups. Both HBI and UC groups had low levels of medication adherence $($ score $<6$ ) and diabetes knowledge scores (score $<7)$ before the intervention. The post intervention results revealed no significant association among demographic and disease-related variables. A statistically significant difference was observed $(p<0.001)$; however, when knowledge, adherence and HbAlc levels were assessed between HBI and UC groups after completion of the intervention. There was an increase in the mean knowledge score (10.04 \pm 1.75$)$ among the HBI group. In addition, medication adherence also improved in the HBI group, with the post-interventional analysis revealing an increase in medication adherence scores $(6.90 \pm 0.94)$. HbAlc was also improved in the HBI group with a difference of 0.73 from the baseline. No noteworthy difference among the UC group in terms of diabetesrelated knowledge, medication adherence and HbA1c levels, compared with baseline analysis, was reported.

The findings of this study have indicated that type 2 diabetes patients in Bukit Minyak clinic were lack of knowledge on the disease and have low level of medication adherence before the Home Medication Review was conducted. The pharmacist-led counseling and education sessions in the intervention group was able to increase the patients' knowledge on the disease and further improve their medication adherence and glycaemic control. Apart from that, this study also supported the role of pharmacist as an educator in reaching out patients beyond healthcare setting rather than a dispenser in the healthcare setting. The pharmacists who could able to spend time with the patients and are more experts in medications could play a better role in the healthcare system. Besides this, the Home Medication Review program was able to build a better relationship between the healthcare providers and the pharmacists with the informal discussion of the pharmaceutical problems. This could have provided an opportunity for the collaboration between the healthcare providers and pharmacists in managing the patients. This study has also provided a clearer picture of the impact of Home Medication Review Program in primary setting for

*Corresponding author: Fahad Saleem, School of Pharmaceutical Sciences, Universiti Sains Malaysia, Malaysia, Tel: 0060124003956; E-mail: fahaduob@gmail.com

Received November 10, 2015; Accepted November 27, 2015; Published December 04, 2015

Citation: Saleem F, Hassali MA, Chow EP (2015) Home Medication Review in Improving Patient Medication Adherence and Minimizing Medication Wastage among Type 2 Diabetes Patients. Health Econ Outcome Res Open Access 1: 104. doi: $10.4172 / 2471-268 x / 1000104$

Copyright: @ 2015 Saleem F, et al. This is an open-access article distributed under the terms of the Creative Commons Attribution License, which permits unrestricted use, distribution, and reproduction in any medium, provided the original author and source are credited. 
Citation: Saleem F, Hassali MA, Chow EP (2015) Home Medication Review in Improving Patient Medication Adherence and Minimizing Medication Wastage among Type 2 Diabetes Patients. Health Econ Outcome Res Open Access 1: 104. doi: 10.4172/2471-268x/1000104

the Ministry of Malaysia Health to formulate better strategies for the implementation in the country.

\section{Reference}

1. David Willens, Rebecca Cripps, Amy Wilson, Kathleen Wolff, Russel Rothman (2011) Interdisciplinary team care for diabetic patients by primary care physicians, advanced practice nurses, and clinical pharmacists. Clinical Diabetes 29: 60-68.
2. Thomas Fiss, Adina Dreier, Claudia Meinke, Neeltje van den Berg, Christoph Alexander Ritter, et al. (2011) Hoffmann Frequency of inappropriate drugs in primary care: analysis of a sample of immobile patients who received periodic home visits. Age and Ageing 40: 66-73.

3. HK Al-Qazaz, MA Hassali, AA Shafie, SAS Sulaiman, S Sundram (2010) The 14-item Michigan Diabetes Knowledge Test: translation and validation study of the Malaysian version. Practical Diabetes International 27: 238-241.

4. Harith Kh Al-Qazaza, Mohamed A Hassalib, Asrul A Shafieb, Syed A Sulaimana, Shameni Sundramc, et al. (2010) The eight-item Morisky Medication Adherence Scale MMAS: Translation and validation of the Malaysian version. Diabetes Research and Clinical Practice 90: 216-221. 